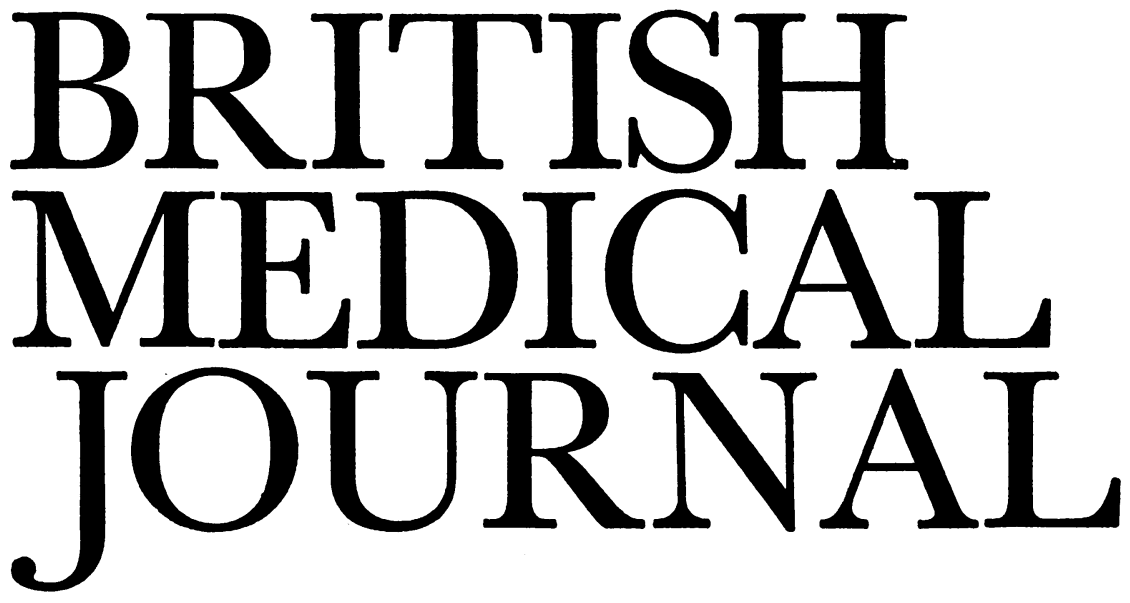

\title{
Irradiation and Leukaemia
}

Since the first report of leukaemia in radiological workers more than 60 years ago abundant evidence has accumulated of the capacity of ionizing radiation to produce leukaemia in man.

In 1957 W. M. Court Brown and R. Doll ${ }^{1}$ reported the first results of their study of a large number of patients with ankylosing spondylitis who had received $x$-ray treatment for this condition during 1935-54. Among this group a highly significant increase in mortality from leukaemia was confirmed. These authors later published additional data, ${ }^{2}$ and by the end of 196260 cases of leukaemia had developed among the 14,554 patients under study. In addition there was an excess of cases of aplastic anaemia, many of which are likely to have been aleukaemic leukaemia. The estimated excess mortality from leukaemia which was attributable to $x$-ray therapy was about 4 per 1,000 irradiated men over a follow-up period averaging 13 years from the first treatment. It was noted that the leukaemia was usually acute and was more often of the myeloid type than among unirradiated persons. Only one death was attributed to chronic lymphatic leukaemia, and in that case the clinical and cytological description proved to be mistaken. The mortality from leukaemia reached a maximum at three to five years after irradiation and then declined.

In the case of patients who had received spinal irradiation alone it was possible to study the relationship between radiation dose and the incidence of leukaemia with some confidence, since the distribution of dose in the treated marrow was accurately known. The relationship was found to be linear, and the size of the risk was estimated to be 1.3 cases per rad of whole-body irradiation per million persons per year, averaged over the seven years after exposure. ${ }^{3}$ In all of these patients the dose of radiation was spread out in fractions over several weeks and often over two or more courses. Relatively few patients were treated with a mean marrow dose of less than 250 rads, so that this study provided no direct evidence of a leukaemogenic effect with low doses of radiation such as are employed in diagnostic radiology or are received from background radiation. The evidence, however, favoured a linear relationship between the dose received and the incidence of leukaemia, suggesting that no threshold existed below which no effect would be produced.

The first definite evidence that diagnostic $x$-rays could be leukaemogenic came from the study by Alice Stewart and her colleagues ${ }^{4}$ of 1,416 children who had died of leukaemia or cancer before their tenth birthday and a similar number of controls. The difference in the frequency of diagnostic abdominal radiographs of the mothers during pregnancy in the case and the control groups indicated that children who had been $x$-rayed in utero were approximately twice as likely to die of a malignant disease before their tenth birthday as other children. It was further estimated that between $6 \%$ and $7 \%$ of all such deaths were caused by diagnostic irradiation during pregnancy.

The explosion of atomic bombs in Japan in 1945 provided an opportunity for investigating the effects of a single exposure to whole-body irradiation in dose-specific terms, and much work by the Atomic Bomb Casualty Commission has gone into estimating the dose received by the survivors. It has been established ${ }^{5}$ that the risk of leukaemia is appreciably increased for people exposed even to 20-50 rads in Hiroshima, though in Nagasaki no increase was found below 200 rads. There were, however, important differences between the two cities in the type of radiation released: the Hiroshima bomb emitted both gamma rays and neutrons, whereas the Nagasaki bomb produced mainly gamma rays. The most probable explanation for the greater risk of leukaemia in Hiroshima than in Nagasaki at every level of radiation exposure is a greater leukaemogenic capacity of neutrons than of gamma rays. Myeloid leukaemia, both acute and chronic, and acute lymphatic leukaemia were all increased among survivors who received an estimated 100 rads or more, the risk of acute lymphatic leukaemia being particularly increased among those under 15 years old at the time of the bombing. The risk of leukaemia declined over the period 1950-66 but was still raised among survivors from the high-dose regions of the cities even 15 to 21 years after the explosions. In neither city was there any evidence of an increase in chronic lymphatic leukaemia. For all years between 1950 and 1966 the best estimate of the effect of irradiation, on the assumption that neutrons were five times as effective as gamma rays, 
was that $1 \mathrm{rad}$ of irradiation produced approximately 1.6 cases of leukaemia per million persons per year.

Thus a clear link has been established between leukaemia and irradiation from large-scale studies of $x$-ray therapy, of diagnostic radiography during pregnancy, and of exposure to the atomic explosions in Japan. Because of the considerable methodological problems it is not surprising that the effects of diagnostic $x$-rays in adults have been studied only to a limited extent. In 1962 Stewart and her colleagues ${ }^{6}$ presented details of previous $x$-ray examinations in 963 cases of adult leukaemia, 60 of lymphosarcoma, 951 of other cancers, and 974 controls. The data were examined in sets of three (leukaemia, cancer, control), each member of the trio being matched for age, sex, district, and interviewer. An excess of radiographs of the trunk was noted in the eight years before the diagnosis of myeloid leukaemia. No excess was noted in lymphatic leukaemia, and it was estimated that about $8 \%$ of leukaemias other than the lymphatic type in adults were caused by diagnostic $x$-rays.

A further study has now been reported ${ }^{7}$ in which previous diagnostic irradiation in 1,414 cases of adult leukaemia (of which 590 were myeloid or monocytic) was compared with that in 1,370 adults selected as controls from the same parts of New York State, Minneapolis, and Baltimore, as the cases. Details of $x$-ray exposure were obtained by more thorough methods than in the previous study, information from the patients or their relatives being supplemented by a search of the records of all physicians and hospitals attended by the patients. Diagnostic $x$-rays, particularly those of the trunk, were found to increase the risk of both acute and chronic myeloid leukaemia in males but not in females. The relative risk of the chronic type for males exposed to 11 or more trunk $x$-rays rose from $2 \cdot 22: 1$ to 7-14:1 for exposures of 41 or more. The corresponding risks for acute myeloid-monocytic leukaemia were $1.36: 1$ and $5.06: 1$ respectively. It was estimated that $8.8 \%$ of cases of chronic myeloid leukaemia in males might be attributable to exposure to 21 or more diagnostic $x$-ray examinations.

This finding of a sex difference in the effects of radiation is in keeping with the greater increase in incidence in males than in females among the survivors of the atomic explosions in Japan, especially in the high-dose groups. No such difference was reported by Stewart and her colleagues in their study of adult leukaemias, while there were too few females with ankylosing spondylitis in Court Brown and Doll's study for a reliable comparison to be made. On present evidence the finding appears real, though unexplained. In this respect it is of interest that in one strain of mices the males developed a higher incidence of leukaemia than the females after both had received the same amounts of irradiation.

The two studies by Stewart and Gibson and their colleagues suggest that, though diagnostic irradiation may be responsible for only a small proportion of all chronic myeloid leukaemia, nevertheless the actual number of cases is large enough to be important.

\footnotetext{
1 Court Brown, W. M., and Doll, R., Medical Research Council Special Reports Series, No. 295. London, H.M.S.O., 1957

2 Court Brown, W. M., and Doll, R., British Medical fournal, 1965, 2, 1327.

International Commission on Radiological Protection, I.C.R.P. Publication 8, The Evaluation of Risks from Radiation. Oxford, Pergamon Press, 1966.

4tewart, A., Webb, J., and Hewitt, D., British Medical fournal, 1958, 1, 1495.

5Ishimaru, T., et al., Radiation Research, 1971, 45, 216.

- Stewart, A., Pennybacker, W., and Barber, R., British Medical fournal, $1962,2,882$.

7 Gibson, R., et al., fournal of the National Cancer Institute, 1972, 48, 301.

8 Upton, IT. C., National Cancer Institute Monographs, 1964, 14, 221.
}

\section{Immunochemotherapy of Cancer}

The use of non-specific cytotoxic drugs in the treatment of patients with cancer has the intrinsic drawback that normal cells in the process of dividing are as susceptible to their lethal action as dividing cells in cancers. Cell proliferation in tissues such as the bone marrow and intestinal mucosa may be more active than in cancerous tissues, and the need to preserve the vital tissues reduces the maximum tolerated doses of drugs of this kind to below those necessary to destroy the cancer.

The possibility of overcoming this limitation by designing cytotoxic drugs that act specifically on cancer cells has inspired a variety of research approaches during recent years. Thus, attempts have been made to prepare cytotoxic drugs with short half-lives suitable for direct injection into arteries supplying cancers, in the hope that no drug would spill over from the area of the cancer to destroy normal tissues. Another idea has been to exploit the low $\mathrm{pH}$ which is characteristic of certain neoplasms as a consequence of the accumulation of lactic acid by glycolysis. ${ }^{1}$ Similarly, the existence of differences in oxidation potential between normal and neoplastic cells has led to attempts to develop tumourspecific cytotoxic drugs. ${ }^{2}$ The possibility of attaching cytotoxic drugs to chemicals required more by tumour cells than by normal cells has been explored in several ways. But none of these efforts has been rewarded by outstanding clinical success.

A serious difficulty may be that no two cases of cancer are exactly the same, for each case of cancer is an expression of a war between a uniquely individual patient and a uniquely individual cancer. An important part of the individuality of both the patient and his cancer is expressed in terms of antigenic composition. The report by Professor $T$. Ghose and his colleagues elsewhere in this issue of the B.M.7. (page 495) describes attempts to exploit the antigenic specificity of a particular type of cancer by attaching a cytotoxic drug to the corresponding tumour-specific antibody.

Firstly, they report the complete regression of a transplanted tumour in a genetically pure strain of mice in response to treatment with a cytotoxic drug chlorambucil bound to tumour-specific antibody. In the same circumstances untreated mice all died, and treatment with unbound chlorambucil, with tumour-specific antibody alone, or with chlorambucil bound to normal rabbit globulin did not inhibit the tumours. Secondly, they record the long and complex history of a patient with disseminated melanoma who, having ceased to respond or having failed to respond at all to a variety of other treatments, showed a distinctly favourable treatment with anti-melanoma globulin combined with chlorambucil.

Unquestionably the report by Professor Ghose and his colleagues is important and it will stimulate many to try to confirm the value of the approach. But it is certainly too early to know whether their findings are destined to revolutionize the prospects of patients with malignant melanoma, or to know too whether they have opened the way to the development of new and more successful methods of treating patients with cancers of other kinds. As the authors themselves point out, malignant melanomas figure prominently in the list of neoplasms for which there are reports of so-called "spontaneous regression." Many such cases are 